\title{
Dispersion due to molecular diffusion and macroscopic mixing in flow through a network of capillaries
}

\author{
By P. G. SAFFMAN \\ Cavendish Laboratory, Cambridge
}

(Received 27 May 1959)

\begin{abstract}
This paper is concerned with the dispersion of a material quantity in the steady flow of a viscous fluid through a random network of capillaries (which is a useful model of a porous medium), for the case in which molecular diffusion and macroscopic mixing, due to the randomness of the streamlines, are both important. A Lagrangian correlation function is introduced and the longitudinal and lateral effective diffusivities are thereby calculated for all values of $U l / \kappa$ less than some large value. Here, $l$ denotes the length of a capillary, $U$ the mean velocity of the fluid, and $\kappa$ the molecular diffusivity of the material quantity. The theory is compared with experimental observations of dispersion in flow through granular beds.
\end{abstract}

\section{Introduction}

In a recent paper (hereafter referred to as I), Saffman (1959) has investigated the dispersion of a dynamically neutral material quantity in a viscous fluid flowing through a porous medium, on the basis that the porous medium may be regarded as equivalent to a statistically isotropic network of straight capillaries. The discussion was restricted to the case in which the dispersion is primarily due to macroscopic mixing. This arises from the randomness of the streamlines through the network, and has an effective diffusivity of order $U l$, where $l$ is the length scale of the capillaries and $U$ is the mean velocity of the fluid (the interstitial velocity). The results of $I$ are then only valid when

$$
\kappa \ll U l,
$$

where $\kappa$ is the molecular diffusivity of the material quantity.

The method of I utilized the fact that the path of a fluid particle through a random network of capillaries can be treated as a random walk of randomly orientated steps, the velocity in a step depending upon its direction. This approach is clearly valid only if (1.1) is satisfied. An alternative approach, involving the idea of Lagrangian correlation functions which are well known in the theory of turbulent diffusion (see $\$ 3$ below), was suggested to the author by $\operatorname{Dr}$ T. H. Ellison in a private communication. As it happens, this method seems to be in general (although not always $\dagger$ ) less useful than the random walk approach for the cases

+ For example, it gives the dispersion in certain cases for small values of the time from the initial instant, whereas the random walk approach is an asymptotic theory for large values of this time. 
considered in I. However, it affords a means of calculating the dispersion when (1.1) is not satisfied, i.e. when the effect of molecular diffusion is comparable with or greater than the macroscopic mixing, and this is the purpose of the present paper. The theory is not without practical importance since a network of capillaries is not an altogether unreasonable model of a porous medium and actual flows in which $U l / \kappa \leqslant O(1)$ are not uncommon, e.g. the flow of gases through porous media.

\section{The flow in the network}

The work is restricted to the case in which the mean (macroscopic) velocity in the network is uniform and unidirectional, and the Reynolds number of the flow in the capillaries is sufficiently small for viscous effects to be dominant (this is equivalent to supposing that the mean flow satisfies Darcy's law). We also suppose (primarily for simplicity, as in $I$ ) that the pores are circular of radius $a$ and of equal length $l$, where $a \ll l$. Then to a reasonable approximation, the average velocity in a capillary making an angle $\theta\left(0<\theta<\frac{1}{2} \pi\right)$ with the direction of mean motion may be taken as

$$
q=3 U \cos \theta,
$$

where $U$ is the average velocity of the entire fluid (see $I$, equation (2.19), where $q$ is denoted by $\hat{q}$ ).

Since this paper is primarily concerned with the dispersion when $\kappa$ is comparable with or greater than $U l$, we shall suppose further that

$$
\frac{U l}{\kappa} \ll \frac{8 l^{2}}{a^{2}} \quad \text { or } \quad \frac{l}{U} \gg \frac{a^{2}}{8 \kappa} .
$$

Now $a^{2} / 8 \kappa$ is the time scale for molecular diffusion to smooth out variations in concentration across the cross-section of a capillary, and the condition (2.2) expresses the condition that this is small compared with the time spent by a fluid particle in a capillary. The work of Taylor $(1953 ; 1954)$ and Aris (1956) shows that in this case the concentration is approximately uniform over the cross-section and disperses, relative to axes moving with the velocity $q$, according to a diffusivity

$$
\kappa+\frac{a^{2} q^{2}}{48 \kappa}=D, \text { say. }
$$

Thus, when (2.2) is satisfied, the dispersion in the network is the same as if the velocity in each capillary were uniform throughout it, with the value $q$, and the material is subject to a diffusion process along the capillary with a diffusivity $D$ (which depends upon the direction of the capillary). Note that

$$
\frac{a^{2} q^{2}}{48 \kappa^{2}}<\frac{3}{16} \frac{a^{2}}{l^{2}}\left(\frac{U l}{\kappa}\right)^{2} \ll \frac{3}{2} \frac{U l}{\kappa}
$$

by virtue of (2.2); so that $D \doteqdot \kappa$ if $U l / \kappa$ is not large compared with unity, and for all $U l / \kappa$ if $a<l$. 


\section{The Lagrangian correlation function}

If $u(t)$ is the velocity component in the $x$-direction of a marked particle participating in a random process, the Lagrangian correlation function or covariance of the velocity $u$ at time $t$ and at a later time $t^{\prime}$ is $\overline{u(t) u\left(t^{\prime}\right)}$, where the bar denotes an average taken in an appropriate way (which will depend upon the precise nature of the random process). As was first pointed out by Taylor (1921), the displacement in the $x$-direction after time $t$ is $X(t)=\int_{0}^{t} u\left(t^{\prime}\right) d t^{\prime}$, and $d X / d t=u(t)$. Hence

$$
\frac{d}{d t} \overline{X^{2}(t)}=2 \overline{X(t) u(t)}=2 \int_{0}^{t} \overline{u(t) u\left(t^{\prime}\right)} d t^{\prime} .
$$

For the case in which $u(t)$ is a stationary random function of the time (e.g. turbulent diffusion along a pipe; see Batchelor \& Townsend 1956), $\overline{u(t) u\left(t^{\prime}\right)}=R\left(t-t^{\prime}\right)$, where $R(\tau)$ is the correlation function of the velocity $u$ for a time interval $\tau$ (and depends only on $\tau$ and not on the time from any given instant), and the axes are chosen so that $\bar{u}=\bar{X}=0$. The overbar here denotes an ensemble or stochastic average. Then (3.1) becomes

$$
\frac{d \overline{X^{2}}}{d t}=2 \int_{0}^{t} R(\tau) d \tau
$$

and hence

$$
\overline{X^{2}}=2 \int_{0}^{t}(t-\tau) R(\tau) d \tau
$$

Thus when $t$ is large compared with the time scale for which the velocities at successive times are effectively correlated,

$$
\overline{X^{2}} \sim 2 t \int_{0}^{\infty} R(\tau) d \tau-2 \int_{0}^{\infty} \tau R(\tau) d \tau,
$$

assuming that the integrals converge.

Thus,

$$
\overline{\overline{X^{2}}} \sim \int_{0}^{\infty} R(\tau) d \tau,
$$

and hence $\int_{0}^{\infty} R(\tau) d \tau$ may beregarded as the effectivediffusivity. Itshould perhaps be pointed out for the benefit of readers unfamiliar with this analysis that (3.4) does not prove that the dispersion of a cloud of marked particles is given (asymptotically) by solutions of the diffusion equation with this effective diffusivity. To do this, it is necessary to prove that the probability distribution of $X$ is normal, and that the displacement of different fluid particles in the same realization of the flow become statistically uncorrelated. However, there are usually no reasons for believing that these requirements are not satisfied, even though in most cases the formulation of rigorous proofs appears to present great difficulties:

If now $u(t)$ denotes the longitudinal component (i.e. in the direction of mean flow) of the velocity of a marked particle in steady flow through a random network of capillaries, we may proceed in an identical manner, provided the network is 
statistically homogeneous. For convenience, we refer $u$ and $X$ to axes moving with the mean velocity, so that

$$
u=\left(q+q_{D}\right) \cos \theta-U=U\left(3 \cos ^{2} \theta-1\right)+q_{D} \cos \theta
$$

where $\theta=\theta(t)$ refers to the capillary containing the particle at time $t . q_{D}$ is the random part of the velocity along the capillary due to the diffusion process with diffusivity $D$; it can be regarded as the velocity due to a Brownian motion equivalent to the diffusion process. That is, the velocity of a marked particle along a capillary fluctuates randomly because of the actual Brownian motion (caused by the collisions with other molecules) and because, as it moves to and fro across the capillary cross-section, the local mean velocity varies. These effects are mathematically equivalent to diffusion with diffusivity $D$ and a corresponding fictitious Brownian velocity $q_{D}$ with the properties

$$
\left\langle q_{D}\right\rangle=0 \text { and } \int_{0}^{\infty}\left\langle q_{D}(0) q_{D}(\tau)\right\rangle d \tau=D
$$

where $\langle>$ denotes an average with respect to the Brownian motion.

This argument is, of course, valid only if the time interval over which successive values of $q_{D}$ are correlated is small compared with the average time spent by a marked particle in a capillary. The former is at most $a^{2} / 8 \kappa$ (and will be much less if molecular transport dominates the diffusion process), whereas the order of magnitude of the latter is the lesser of $l / U$ and $l^{2} / 2 \kappa$, and the argument is therefore valid by virtue of (2.2) and the condition $a \ll l$.

For the case of turbulent flow, the average denoted by the overbar is an ensemble average over all realizations of the flow. For the case of steady flow in a random network, the average is over all configurations of the network, but this is equivalent, owing to the statistical homogeneity of the network, to an average over the initial position and velocity of the particle. Thus, if $f$ denotes a property of a marked particle which at time $t=0$ was in a pore making an angle $\theta\left(0<\theta<\frac{1}{2} \pi\right)$ with the direction of mean motion and with azimuthal angle $\phi(0<\phi<2 \pi)$ about the same direction, was at a distance $l \xi(0<\xi<1)$ from the entrance of the capillary, and had a 'Brownian velocity' $q_{D}$, where the values of $\xi, \phi$ and $\mu=\cos \theta$ are uniformly distributed (since the proportion of pores in the range $\theta$ to $\theta+d \theta$ is $\sin \theta d \theta$ ), then

$$
\bar{f}=\int_{0}^{\frac{1}{2 \pi}} \int_{0}^{1} \int_{0}^{2 \pi} \int_{0}^{1} f \sin \theta d \theta d \xi \frac{d \phi}{2 \pi} d p\left(q_{D}\right),
$$

where $p\left(q_{D}\right)$ is the probability that the 'Brownian velocity' is less than $q_{D}$. Note that

$$
\langle f\rangle=\int_{0}^{1} f d p\left(q_{D}\right)
$$

The value of $\bar{f}$ is independent of the precise origin of the time. Moreover, because of the statistical homogeneity, $\overline{u(t)}=\overline{u(0)}=0$, and $\overline{u(t) u\left(t^{\prime}\right)}$ is a function of $\left(t^{\prime}-t\right)$ only, equal to $R\left(t^{\prime}-t\right)$, say. Thus the covariance is given by

$$
R(\tau)=\overline{u(0) u(\tau)},
$$

with the dispersion following from (3.3) and (3.4). 


\section{Calculation of the covariance}

Let primed quantities denote values after a time $\tau$ from some initial instant. We have, writing $\mu=\cos \theta, \mu^{\prime}=\cos \theta^{\prime}$,

$$
\overline{u u^{\prime}}=U^{2} \overline{\left(3 \mu^{2}-1\right)\left(3 \mu^{\prime 2}-1\right)}+\overline{\mu \mu^{\prime} q_{D} q_{D}^{\prime}}+U\left\{\overline{\mu q_{D}\left(3 \mu^{\prime 2}-1\right)+\mu^{\prime} q_{D}^{\prime}\left(3 \mu^{2}-1\right)}\right\} .
$$

The last term in (4.1) is zero since $\left\langle q_{D}\right\rangle=0$ and $\mu$ is independent of $q_{D}$.

Consider now the second term. The time interval over which $q_{D}$ and $q_{D}^{\prime}$ are effectively correlated is small compared with the average time for the particle to leave the initial capillary and enter another. In other words $\left\langle q_{D} q_{D}^{\prime}\right\rangle$ is nonnegligible only for values of $\tau$ so small that the value of $\mu$ does not change (except for a negligible range of values of $\xi$ for which a particle is next to a pore junction). Hence, to a reasonable approximation,

$$
\overline{\mu \mu^{\prime} q_{D} q_{D}^{\prime}}=\overline{\mu^{2} q_{D} q_{D}^{\prime}}=\int_{0}^{1} \mu^{2}\left\langle q_{D} q_{D}^{\prime}\right\rangle d \mu,
$$

on integrating with respect to $\xi$ and $\phi$. (This is permissible since $\left\langle q_{D} q_{D}^{\prime}\right\rangle$ will depend, to the present approximation, only on $\tau$ and the mechanics of the diffusion process with diffusivity $D$.)

It now follows from (3.6) that

$$
\begin{aligned}
\int_{0}^{t} \overline{\mu \mu^{\prime} q_{D} q_{D}^{\prime}} d \tau & =\int_{0}^{1} D \mu^{2} d \mu \quad\left(\text { if } t \gg a^{2} / 8 \kappa\right) \\
& =\frac{\kappa}{3}+\frac{3}{80} \frac{a^{2} U^{2}}{\kappa}
\end{aligned}
$$

on substituting from (2.3) and (2.1).

Thus the problem reduces to the calculation of the first term in (4.1), namely,

$$
\left(\overline{\left.3 \mu^{2}-1\right)\left(3 \mu^{\prime 2}-1\right)}=S(\tau), \quad\right. \text { say. }
$$

There are two distinct contributions to $S(\tau)$. First, there is the integral over those initial conditions such that the marked particle has not reached either of the ends of the initial capillary after time $\tau$, so that $\mu=\mu^{\prime}$; this gives a contribution $S_{1}(\tau)$, say. Secondly, there is the integral over the conditions for which the particle is in a different capillary after time $\tau$ (or has returned to the initial capillary after having passed through one of the junctions at its ends), giving a contribution $S_{2}(\tau)$, say. Then $S(\tau)=S_{1}(\tau)+S_{2}(\tau)$.

\subsection{The calculation of $S_{1}(\tau)$}

Let $P\left(\xi_{0}, \mu, \tau\right)$ denote the probability that a marked particle released at time $\tau=0$ at a distance $l \xi_{0}$ from the entrance of the capillary has not reached one of the ends after time $\tau$. Since $\mu=\mu^{\prime}$ for a particle which remains in the initial capillary,

$$
S_{1}(\tau)=\int_{0}^{1} \int_{0}^{1} \int_{0}^{2 \pi}\left(3 \mu^{2}-1\right)^{2} P d \mu d \xi_{0} \frac{d \phi}{2 \pi}
$$

$\left(P\left(\xi_{0}, \mu, \tau\right)\right.$ is in fact the integral of $d p\left(q_{D}\right)$ over those modes of the 'Brownian motion' such that $\xi \neq 0$ or 1 in time $\tau$.) 
To calculate $P$, we note that the marked particles are convected along the capillary with velocity $q(=3 U \mu)$ and diffuse relative to an origin moving at this speed with a diffusivity $D$. If they reach the ends of the capillary, they no longer contribute to $S_{1}(\tau)$. Hence the probability that the particle lies between $l \xi$ and $l(\xi+d \xi)$ after time $\tau$ (not having passed through $\xi=0$ or 1 ) is $c d \xi$, where $c(\xi, \tau)$ is the solution of

$$
\frac{\partial c}{\partial \tau}+\frac{q}{l} \frac{\partial c}{\partial \xi}=\frac{D}{l^{2}} \frac{\partial^{2} c}{\partial \xi^{2}}
$$

subject to the initial condition $c=\delta\left(\xi-\xi_{0}\right)$ when $\tau=0(\delta(\xi)$ is the Dirac delta function) and the boundary conditions $c=0$ when $\xi=0$ and 1 for all $\tau$. Then

$$
P\left(\xi_{0}, \mu, \tau\right)=\int_{0}^{1} c d \xi
$$

The solution of (4.4) is straightforward. It is easily verified that

where

$$
\begin{gathered}
C=\sum_{1}^{\infty} a_{n} \sin n \pi \xi e^{M \xi} \exp \left\{-\left(n^{2} \pi^{2}+M^{2}\right) D \tau / l^{2}\right\}, \\
M=\frac{q l}{2 D}=\frac{\frac{3}{2} U l \mu}{\kappa+3 a^{2} U^{2} \mu^{2} / 16 \kappa},
\end{gathered}
$$

is a solution of the differential equation which satisfies the boundary conditions. It satisfies the initial conditions if

$$
\delta\left(\xi-\xi_{0}\right)=\sum_{1}^{\infty} a_{n} \sin n \pi \xi e^{M / \xi},
$$

from which it follows by the usual methods of Fourier series that

Then (4.5) gives

$$
a_{n}=2 e^{-M \xi_{0}} \sin n \pi \xi_{0} \text {. }
$$

$$
P\left(\xi_{0}, \mu, \tau\right)=\sum_{1}^{\infty} \frac{2 n \pi}{n^{2} \pi^{2}+M^{2}}\left\{1-(-1)^{n} e^{M}\right\} e^{-M \xi_{0}} \sin n \pi \xi_{0} \exp \left\{-\left(n^{2} \pi^{2}+M^{2}\right) D \tau / l^{2}\right\} .
$$

On substituting in (4.3) and integrating with respect to $\xi_{0}$ and $\phi$, we obtain

$$
\begin{aligned}
S_{1}(\tau)=\int_{0}^{1} 4 d \mu\left(3 \mu^{2}-1\right)^{2} & \sum_{1}^{\infty} \frac{n^{2} \pi^{2}}{\left(n^{2} \pi^{2}+M^{2}\right)^{2}} \\
& \times\left\{1-(-1)^{n} \cosh M\right\} \exp \left\{-\left(n^{2} \pi^{2}+M^{2}\right) D \tau / l^{2}\right\},
\end{aligned}
$$

where, in general, $D$ and $M$ are functions of $\mu=\cos \theta$.

This expression is fairly complicated. However, for the purpose of finding the effective diffusivity we only require the value of $\int_{0}^{\infty} S_{1}(\tau) d \tau$. Integrating (4.8) term by term and summing the infinite series, we find

$$
\begin{aligned}
\int_{0}^{\infty} S_{1}(\tau) d \tau & =\int_{0}^{1} 4 d \mu\left(3 \mu^{2}-1\right)^{2} \sum_{1}^{\infty} \frac{l^{2}}{D} \frac{n^{2} \pi^{2}}{\left(n^{2} \pi^{2}+M^{2}\right)^{3}}\left\{1-(-1)^{n} \cosh M\right\} \\
& =\int_{0}^{1} d \mu \frac{l^{2}}{4 D}\left(3 \mu^{2}-1\right)^{2}\left(\frac{1}{M} \operatorname{coth} M-\frac{1}{M^{2}}\right) .
\end{aligned}
$$


We have here made use of the following formulae (which may be obtained by expressing $\cosh \lambda x$ as a Fourier cosine series in $x$ and putting $x=0$ and $\pi$ ):

$$
\begin{gathered}
\frac{\cosh \lambda-1}{2 \lambda^{2}}=\sum_{1}^{\infty} \frac{1-(-1)^{n} \cosh \lambda}{n^{2} \pi^{2}+\lambda^{2}}, \\
\frac{\lambda-\sinh \lambda}{2 \lambda^{2} \sinh \lambda}=\sum_{1}^{\infty} \frac{(-1)^{n}}{n^{2} \pi^{2}+\lambda^{2}},
\end{gathered}
$$

and the formulae which can be obtained by differentiating them with respect to $\lambda$.

\subsection{The calculation of $S_{2}(\tau)$}

The determination of $S_{2}(\tau)$ is rather more difficult. We shall assume, first, that the values of $\mu$ for successive capillaries are statistically independent; that is, when a particle reaches a junction the choice of the next capillary is independent of the previous one. The larger $\kappa / U l$ the better will be this assumption, because molecular diffusion has a much smaller correlation time scale than the macroscopic mixing.

It might now be thought that

$$
S_{2}(\tau)=\left[\left(\overline{\left.3 \mu^{2}-1\right)\left(3 \mu^{\prime 2}-1\right)}\right]_{\mu \neq \mu^{\prime}}=\overline{\left(3 \mu^{2}-1\right)} \overline{\left(3 \mu^{\prime 2}-1\right)}=0 .\right.
$$

However, this argument is false because it ignores the fact that although $\mu^{\prime}$ is independent of $\mu$, it is not independent of the fact that the particle has passed through a junction during the time $\tau$ and this, as will be seen below, affects the probability distribution of $\mu^{\prime}$. Nevertheless, it is in fact true that $\int_{0}^{\infty} S_{2}(\tau) d \tau=0$, so that $S_{2}(\tau)$ does not contribute to the effective diffusivity. $\int_{0}^{\infty} \tau S_{2}(\tau) d \tau \neq 0$, so there is a contribution to $\overline{X^{2}}$. The remainder of this section is devoted to the calculation of $S_{2}(\tau)$, or, to be more precise, its Laplace transform which is sufficient for our purposes.

Let $Q(\mu, t) d \mu$ denote the probability that a marked particle released at a junction at $t=0$ is in a capillary in the range $\mu$ to $\mu+d \mu$ after time $t$.

The probability that a particle released at $t=0$ at a distance $l \xi$ from the entrance to a capillary with a given value of $\mu$ reaches a junction in the time interval from $t$ to $t+d t$ is

$$
\frac{\partial}{\partial t}\{1-P(\xi, \mu, t)\} d t
$$

where $P(\xi, \mu, t)$ is given by (4.7). Hence the probability that this particle is in a capillary in the range $\mu^{\prime}$ to $\mu^{\prime}+d \mu^{\prime}$ after time $\tau$ from the initial instant of release is

$$
d \mu^{\prime} \int_{0}^{\tau} Q\left(\mu^{\prime}, \tau-t\right) \frac{\partial}{\partial t}\{1-P(\xi, \mu, t)\} d t=L\left(\xi, \mu, \mu^{\prime}, \tau\right) d \mu^{\prime}, \quad \text { say. }
$$

Then by definition

$$
S_{2}(\tau)=\int_{0}^{1} \int_{0}^{1} \int_{0}^{1} d \xi d \mu d \mu^{\prime}\left(3 \mu^{2}-1\right)\left(3 \mu^{\prime 2}-1\right) L\left(\xi, \mu, \mu^{\prime}, \tau\right) .
$$


If all the pores leading from a junction had equal probability of being entered by a marked particle, then $Q(\mu, t)=1$ and $S_{2}(\tau) \equiv 0$. However, this is not the case since there is a tendency for particles to enter pores in which $q$ is large rather than those in which it is small. (If $\kappa \ll U l$, the probability of a particle leaving a junction by a pore in the range of width $d \mu$ is $2 \mu d \mu$, and $Q(\mu, t)=2 \mu$ for $t \ll l U$.) On the other hand, for large values of $t$ the particle will (so to speak) forget where it was at $t=0$ and the probability of the particle being in a capillary in a particular direction will become independent of the direction, i.e. $Q(\mu, t) \rightarrow 1$ as $t \rightarrow \infty$. But since $S_{2}(\tau)$ depends upon the values of $Q(\mu, t)$ for all values of $t$ between 0 and $\tau$, $S_{2}(\tau)$ will not in general vanish.

It is useful now to take Laplace transforms with respect to the time. These will be denoted by an asterisk, i.e. the transform of $f(t)$ is $f^{*}(p)$. Then since (4.10) is a convolution, we have on integrating with respect to $\xi$,

where

$$
S_{2}^{*}(p)=\int_{0}^{1} \int_{0}^{1} d \mu d \mu^{\prime}\left(3 \mu^{2}-1\right)\left(3 \mu^{\prime 2}-1\right) Q^{*}\left(\mu^{\prime}, p\right)\left\{1-p P_{1}^{*}(\mu, p)\right\}
$$

Now for the effective diffusivity, we really require only $\int_{0}^{\infty} S_{2}(\tau) d \tau$ and this is $S_{2}^{*}(O)$. It now follows from (4.11) and (4.12) that $S_{2}^{*}(O)=0$, provided

$$
\lim _{p \rightarrow 0} \int_{0}^{1}\left(3 \mu^{\prime 2}-1\right) Q^{*}\left(\mu^{\prime}, p\right) d \mu^{\prime}
$$

is finite. We know intuitively that $Q\left(\mu^{\prime}, t\right) \rightarrow 1$ as $t \rightarrow \infty$, and if we assume that $Q\left(\mu^{\prime}, t\right)-1$ is integrable from 0 to $\infty$, it follows that $Q^{*}\left(\mu^{\prime}, p\right)=p^{-1}+O(1)$ as $p \rightarrow 0$, and hence that (4.14) is finite. However, this last assumption is far from obvious $\left(Q\left(\mu^{\prime}, t\right)-1\right.$ might be $\left.O(1 / t)\right)$ and it therefore seems worth while presenting an explicit calculation of $Q^{*}\left(\mu^{\prime}, p\right)$.

Let $E\left(\mu^{\prime}, t\right) d \mu^{\prime}$ denote the probability that a particle released at a junction is after time $t$ in a capillary leading from this junction in the range $\mu^{\prime}$ to $\mu^{\prime}+d \mu^{\prime}$, and in this time $t$ has not returned to the junction or gone to the other end of the pore. The value of $\mu^{\prime}$ in (and only in) $E\left(\mu^{\prime}, t\right)$ ranges from -1 to +1 (elsewhere $0 \leqslant \mu^{\prime} \leqslant 1$ ), to take account of the fact that the particle may diffuse into a capillary against the direction of the stream. Then $Q\left(\mu^{\prime}, t\right)$ is related to $E\left(\mu^{\prime}, t\right)$ by

$$
Q\left(\mu^{\prime}, t\right)=E\left(\mu^{\prime}, t\right)+E\left(-\mu^{\prime}, t\right)+\int_{0}^{t} d t^{\prime} Q\left(\mu^{\prime}, t-t^{\prime}\right) \frac{\partial}{\partial t^{\prime}}\left[1-\int_{-1}^{+1} E\left(\mu, t^{\prime}\right) d \mu\right]
$$

the last term being the probability that it was at a junction sometime during the interval 0 to $t$ and went on from there into a pore with $\mu^{\prime}$ in the appropriate range. Taking Laplace transforms of (4.15), we obtain

$$
\begin{aligned}
& Q^{*}\left(\mu^{\prime}, p\right)=E^{*}\left(\mu^{\prime}, p\right)+E^{*}\left(-\mu^{\prime}, p\right)+Q^{*}\left(\mu^{\prime}, p\right)\left[1-p \int_{-1}^{+1} E^{*}(\mu, p) d \mu\right], \\
& \text { since } \int_{-1}^{+1} E(\mu, 0) d \mu=1
\end{aligned}
$$


Hence

$$
Q^{*}\left(\mu^{\prime}, p\right)=\frac{E^{*}\left(\mu^{\prime}, p\right)+E^{*}\left(-\mu^{\prime}, p\right)}{p \int_{-1}^{+1} E^{*}(\mu ; p) d \mu}
$$

Now $E(\mu, t)$ may be determined in a manner similar to that for $P(\xi, \mu, t)$, except that care is required because the particle starts at a junction. We overcome this difficulty by taking $c=1 / \Delta T$, for $\xi=0$ and $0<t<\Delta T$, as the boundary condition on the concentration $c(\xi, t)$ along the capillary, where $c(\xi, t)$ satisfies (4.4); and $c=0$ initially, $c=0$ at $\xi=1$ for all $t$, and $c=0$ at $\xi=0$ for $t>\Delta T$. Then, we takt

$$
E(\mu, t)=\lim _{\Delta T \rightarrow 0} \int_{0}^{1} c(\xi, t) d \xi, \quad \text { and } E^{*}(\mu, p)=\lim _{\Delta T \rightarrow 0} \int_{0}^{1} c^{*}(\xi, p) d \xi .
$$

Taking the Laplace transform of (4.4), we find that $c^{*}(\xi, p)$ satisfies

$$
\frac{\partial^{2} c^{*}}{\partial \xi^{2}}-2 M \frac{\partial c^{*}}{\partial \xi}-\frac{l^{2} p}{D} c^{*}=0
$$

which has to be solved subject to the boundary conditions

$$
c^{*}(1, p)=0, \quad c^{*}(0, p)=\frac{1}{p \Delta T}\left[1-e^{-p \Delta T}\right] \rightarrow 1 \quad \text { as } \quad \Delta T \rightarrow 0 .
$$

The solution of (4.18) is perfectly straightforward (it is a linear equation with constant coefficients) and we find after a little algebra that

$$
E^{*}(\mu, p)=\frac{M \sinh \left(M^{2}+l^{2} p / D\right)^{\frac{1}{2}}+\left(M^{2}+l^{2} p / D\right)^{\frac{1}{2}}\left\{\cosh \left(M^{2}+l^{2} p / D\right)^{\frac{1}{\frac{1}{2}}}-e^{M}\right\}}{\left(l^{2} p / D\right) \sinh \left(M^{2}+l^{2} p / D\right)^{\frac{1}{2}}}
$$

(4.19), (4.17), (4.13) and (4.12) thus give an explicit expression for $S_{2}^{*}(p)$, and hence determine, in principle, $S_{2}(\tau)$. We find from (4.19) and (4.17) that, as $p \rightarrow 0$,

$$
p Q^{*}(\mu, p)=1+1 l^{2} p\left[\frac{1-M \operatorname{coth} M}{M^{2}}-\int_{0}^{1} d \mu \frac{1-M \operatorname{coth} M}{M^{2} D}\right]
$$

1) is finite and $t$

$$
\int_{0}^{\infty} S_{2}(\tau)
$$

\section{The longitudinal dispersion}

Collecting together the results of $\S 4$, we have from (4.2), (4.9) and (4.21), the result that the longitudinal dispersion in a network of capillaries is asymptotically described by an effective diffusivity

$$
\begin{gathered}
\kappa_{l}=\frac{\kappa}{3}+\frac{3}{80} \frac{a^{2} U^{2}}{\kappa}+\frac{l^{2} U^{2}}{4} \int_{0}^{1}\left(3 \mu^{2}-1\right)^{2} \frac{M \operatorname{coth} M-1}{D M^{2}} d \mu \\
M=\frac{3}{2} \frac{U l \mu}{D} \text { and } D=\kappa+\frac{3 a^{2} U^{2} \mu^{2}}{16 \kappa}
\end{gathered}
$$

where

The expression for $\kappa_{l}$ is valid provided we may replace the upper limit of integration in the expression (3.3) for $\overline{X^{2}}$ by $t=\infty$. This is permissible when the 
exponential term in (4.8) involving the time is small compared with unity and $S_{2}^{*}(1 / t) \div S_{2}^{*}(0)$, which is the case if $t \gg l^{2} / \kappa$.

When there is no convection and no macroscopic mixing (i.e. $U=0$ ), it follows from (5.1) that $\kappa_{l}=\frac{1}{3} \kappa$. Now experiments on dispersion in granular beds (e.g. Carberry \& Bretton 1958) give values of $\kappa_{l} \doteqdot \frac{2}{3} \kappa$, when convection is negligible. The discrepancy seems to be due quite simply to a granular bed not being a random network of capillaries.

However, this is not to say that the present theory is not applicable to dispersion in granular beds; it is at least of qualitative value. Moreover, when there is flow through a granular bed, the streamlines will approximate to those through a random network of capillaries, and it is not too unreasonable to suppose that the macroscopic mixing due to the meanderings of the streamlines is given approximately by the terms in (5.1) involving $U$. In other words, we suggest that (for the purposes of comparison with experiments in granular beds) the above expression for $\kappa_{l}$ be replaced by

$$
\kappa_{l}=\kappa_{m}+\frac{3}{80} \frac{a^{2} U^{2}}{\kappa}+1 l^{2} U^{2} \int_{0}^{1}\left(3 \mu^{2}-1\right)^{2} \frac{M \operatorname{coth} M-1}{D M^{2}} d \mu,
$$

where $\kappa_{m}$ is the measured diffusivity when $U=0$ (and $M$ and $D$ are given by (5.2)). The closer the geometry of the bed approaches that of a random network, then presumably the closer will be $\kappa_{m}$ to $\frac{1}{3} \kappa$. The calculating of $\kappa_{m}$ presents great difficulties; however, it may be readily measured by means of an electrical analogue (see, for example, Wooding 1959).

The expression (5.3) for $\kappa_{l}$ still contains two quantities, $a$ and $l$, whose values have to be fixed in some way from the properties of the granular bed. For $a$, a reasonable value is $a=(24 k / \sigma)^{\frac{1}{2}}$, where $k$ and $\sigma$ are the permeability and porosity, respectively, of the bed. The bed and the random network will then have the same permeability (see I, §2). In any case, the values of $\kappa_{l}$ are fairly insensitive to changes in $a$, provided $a / l$ is not greater than about $\frac{1}{5}$ (which is about the largest permissible value consistent with the assumptions about the flow through the capillaries).

As regards $l$, probably the best value to take is the mean diameter of the grains, and it is not possible to be more precise than this. However, it may be an underestimate, especially if there are cracks or fissures or channels running through the bed. The presence of these would be equivalent to increasing the value of $l$, since they roughly correspond to longer capillaries. We can in fact work out the theory for a network of capillaries of varying lengths; this simply gives (5.1) averaged over the lengths of the cepillaries, but since the distribution of capillary lengths is unknown, there is no real loss in supposing them to be all equal to some suitably chosen value which may be somewhat larger than the mean particle diameter if channels run through the bed or the packing is not too uniform.

The longitudinal diffusivity as given by (5.3) may be evaluated numerically for given values of $a / l$ and $U l / \kappa$. For $U l / \kappa \ll 1$,

$$
\kappa_{l} \doteqdot \kappa_{m}+\frac{1}{15} \frac{U^{2} l^{2}}{\kappa}
$$


and for $U l / \kappa \gg 1$ (but not so large that (2.2) is violated), it can be shown that

$$
\kappa_{l} \doteqdot \frac{U l}{6}\left\{\log _{e} \frac{3}{2} \frac{U l}{\kappa}-\frac{17}{12}-\frac{1}{8} \frac{a^{2}}{l^{2}} \frac{U l}{\kappa}\right\}+\kappa_{m}+\frac{4}{9} \kappa+O\left(\frac{\kappa^{2}}{U l}\right)
$$

The expression (5.5) may be compared with the equivalent expression obtained in $I$ (equations $(4.4 a)$ and (4.5)) by means of the random walk analysis, namely

$$
\kappa_{l} \doteqdot \frac{U l}{6}\left(\log _{e} \frac{3 U l}{2 \kappa}-\frac{1}{4}\right)
$$

The leading terms agree, providing a useful check on the analysis. The difference in the other terms seems to arise from the fact that in I molecular diffusion was taken into account only in an approximate manner.

Hiby (1959) gives the following empirical formula for longitudinal dispersion in beds of glass spheres:

$$
\kappa_{l}=0 \cdot 67 \kappa+\frac{0 \cdot 65 U l}{1+6 \cdot 7(\kappa / U l)^{\frac{1}{2}}},
$$

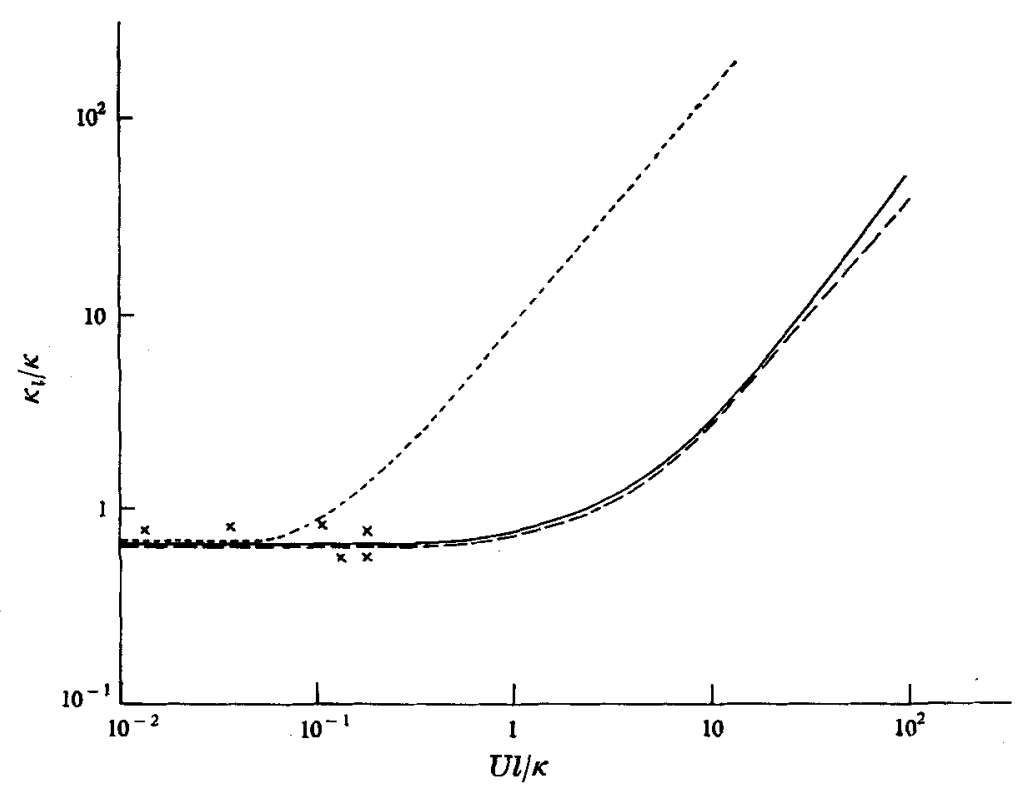

Figure 1. Comparison of theory and experiment for the longitudinal dispersion. _. _., Hiby (1959); ...... Blackwell et al. (1959); $x$, Carberry \& Bretton (1958); - calculated values from (5.3) with $a / l=\frac{1}{5}$ and $\kappa_{m}=\frac{2}{3} \kappa$.

where $l$ is the mean diameter of the spheres. According to this expression, macroscopic mixing is completely negligible for $U l / \kappa<0.5$. On the other hand, Blackwell, Rayne \& Terry (1959) suggest on the basis of experiments in beds of sand the empirical expression:

$$
\frac{\kappa_{l}}{\kappa}=8 \cdot 8\left(\frac{U l}{\kappa}\right)^{1 \cdot 17} \text { for } \frac{U l}{\kappa}>0.5,
$$

where $l$ is the mean particle diameter. Also, they find that macroscopic mixing becomes significant at about $U l / \kappa=0.04$. 
These results are shown on figure 1 , together with the theoretical curve taking $a / l=\frac{1}{5}$ and $\kappa_{m}=\frac{2}{3} \kappa\left(a / l=\frac{1}{10}\right.$ is probably a better value for a bed of sand but the difference in the calculated values is slight). It will be seen that the theory predicts that macroscopic mixing is negligible for $U l / \kappa<0.5$, and the calculated values are in remarkably good agreement with the expression of Hiby. Nevertheless, the discrepancy between the experimental observations is such that it is not possible to say definitely whether or not the theory is valid when applied to granular beds. A possible explanation of the differences might be that the packing of the sand in some of the experiments was not uniform, in particular there may have been gaps or channels along the walls of the cylinders containing the medium. Hiby states that he was unable to obtain uniform packing right up to the walls of his apparatus, and he went to great trouble to eliminate the wall effect which could affect the results to a factor of two or more. As mentioned previously, non-uniform packing or the presence of channels along the walls would be equivalent to an increase in the value of $l$, and would thereby reduce the discrepancy between the calculated values and the empirical curve of Blackwell et al.; but it is not yet possible to say whether this explanation is sufficient.

\section{The skewness of the longitudinal dispersion}

In the previous section, it was tacitly assumed that the longitudinal distribution of concentration was Gaussian, so that it is meaningful to regard $\overline{X^{2}} / 2 t$ as an effective diffusivity. We shall now obtain an estimate of $\overline{X^{3}}$ in order to see how Gaussian the distribution actually is. This calculation is not without practical importance since observed concentration profiles are often skew.

By arguments similar to those in $\$ 3$, it follows that

$$
\begin{aligned}
\frac{d}{d t} \overline{X^{3}}=3 \overline{X^{2}(t) u(t)} & =3 \int_{0}^{t} \int_{0}^{t} \overline{u(t) u\left(t^{\prime}\right) u\left(t^{\prime \prime}\right)} d t^{\prime} d t^{\prime \prime} \\
& =6 \int_{0}^{t} d \tau_{2} \int_{0}^{\tau_{2}} R_{3}\left(\tau_{1}, \tau_{2}\right) d \tau_{1}
\end{aligned}
$$

where

$$
R_{3}\left(\tau_{1}, \tau_{2}\right)=\overline{u(0) u\left(\tau_{1}\right) u\left(\tau_{2}\right)}
$$

is a triple Lagrangian correlation function.

Now there are two types of contribution to $R_{3}\left(\tau_{1}, \tau_{2}\right)$. First, there are the contributions from the cases in which the particle is in the same capillary after time $\tau_{2}$ from the instant of release; and secondly, from the cases in which it is in a different capillary after time $\tau_{2}$. We shall assume that the second contribution has negligible effect on the value of $\overline{X^{3}}\left(t\right.$ ) for large values of $t$ (as is the case for $\overline{X^{2}}$ ). Although this assumption is highly plausible, a rigorous proof seems to present great difficulties.

To calculate the first contribution, we require the probability that a particle released at a given position is in the same capillary after time intervals $\tau_{1}$ and $\tau_{2}$, where $\tau_{1}<\tau_{2}$, and the particle has not been to one of the ends of the capillary and 
come back again (this is counted as being in a different pore). This probability is just $P\left(\xi_{0}, \mu, \tau_{2}\right)$, as given by (4.7). Hence, for $\tau_{1}<\tau_{2}$,

$$
\begin{aligned}
R_{8}\left(\tau_{1}, \tau_{2}\right)= & U^{3} \int_{0}^{1} \int_{0}^{1}\left(3 \mu^{2}-1\right)^{3} P\left(\xi_{0}, \mu, \tau_{2}\right) d \xi_{0} d \mu \\
= & 4 U^{3} \int_{0}^{1}\left(3 \mu^{2}-1\right)^{3} d \mu \sum_{1}^{\infty} \frac{n^{2} \pi^{2}}{\left(n^{2} \pi^{2}+M^{2}\right)^{2}} \\
& \quad \times\left\{1-(-1)^{n} \cosh M\right\} \exp \left\{-\left(n^{2} \pi^{2}+M^{2}\right) D \tau / l^{2}\right\},
\end{aligned}
$$

since the contribution from the 'Brownian velocity' vanishes. Then

$$
\begin{aligned}
\overline{X^{3}} \sim t & \int_{0}^{\infty} d \tau_{2} \int_{0}^{r_{2}} R_{3} d \tau_{1} \\
& =4 U^{3} t \int_{0}^{1}\left(3 \mu^{2}-1\right)^{3}\left(\frac{l^{2}}{D}\right)^{2} \sum_{1}^{\infty} \frac{n^{2} \pi^{2}}{\left(n^{2} \pi^{2}+M^{2}\right)^{4}}\left\{1-(-1)^{n} \cosh M\right\} d \mu \\
& =\frac{U^{3} l^{4} t}{24} \int_{0}^{1}\left(3 \mu^{2}-1\right)^{3} \frac{1}{D^{2}}\left\{\frac{1}{M^{2}}+\frac{3}{M^{2} \sinh ^{2} M}-\frac{3}{M^{4}}\right\} d \mu .
\end{aligned}
$$

If $U l / \kappa \ll 1$, then

$$
\overline{X^{3}} \doteqdot \frac{U^{3} t l^{4}}{120 \kappa^{2}}
$$

and if $U l / \kappa \gg 1$, it can be shown that

$$
\overline{X^{3}} \div-\frac{U^{2} t l^{3}}{40 K}
$$

(An estimate of $\overline{X^{3}}$ for large values of $U l / K$ is given in equation (3.20) of I. The expressions are the same, except for the numerical coefficient.)

It is clear that $\overline{X^{3}}$ increases linearly with $t$ and in this sense the distribution becomes more skew as the time increases. However, the skewness factor $\overline{X^{3}} /\left(\overline{X^{2}}\right)^{\frac{3}{2}}$ tends to zero like $t^{-\frac{1}{2}}$, and since there is no reason to doubt that the higher moments tend to those of a Gaussian distribution as $t^{-1} \rightarrow 0$ (a rigorous proof of this statement is difficult and will not be attempted here), we may say that the distribution is asymptotically Gaussian.

A measure of the departure from normality is given by the value of the skewness factor. If $U l / \kappa<O(1)$, the skewness factor is always small. The more interesting case is when $U l / \kappa \gg 1$. The condition that the skewness factor be small compared with unity is then

$$
\left(\frac{U t}{l}\right)^{\frac{1}{2}} \gg \frac{U l}{20 \kappa}\left(\log \frac{3 U l}{2 \kappa}\right)^{-\frac{3}{2}}
$$

(This condition is similar to that given in I, equation (4.4a), but it is a little less restrictive.)

\section{Lateral dispersion}

The dispersion in a fixed direction perpendicular to the mean velocity can be calculated in a similar manner as that in the longitudinal direction. We take $y$ and $z$-axes perpendicular to the mean velocity, and denote by $\phi$ the azimuthal angle between the $y$-axis and the projection of the capillary on the $y z$-plane. That 
is, if $q$ is the velocity of fluid in a capillary, the components of velocity parallel to the axes are $q \cos \theta, q \sin \theta \cos \phi, q \sin \theta \sin \phi$. It follows from the assumption of statistical isotropy that all values of $\phi(0 \leqslant \phi<2 \pi)$ are equally likely.

The velocity of a particle in the $y$-direction is

$$
v(t)=3 U \cos \theta \sin \theta \cos \phi+q_{D} \sin \theta \cos \phi,
$$

and the lateral diffusivity is

We have, as in $\$ 4$,

$$
\kappa_{\imath}=\int_{0}^{\infty} \overline{v(0) v(\tau)} d \tau
$$

$$
\overline{v v^{\prime}}=9 U^{2} \overline{\cos \theta \sin \theta \cos \phi \cos \theta^{\prime} \sin \theta^{\prime} \cos \phi^{\prime}}+\overline{q_{D} q_{D}^{\prime} \sin ^{2} \theta \cos ^{2} \phi} .
$$

The last term on the right-hand side of (7.3) makes a contribution to $\kappa_{t}$ of amount

Now to calculate

$$
\begin{aligned}
\overline{D \sin ^{2} \theta \cos ^{2} \phi} & =\frac{\kappa}{3}+\frac{3 a^{2} U^{2}}{16 \kappa} \int_{0}^{1} \mu^{2}\left(1-\mu^{2}\right) d \mu \\
& =\frac{\kappa}{3}+\frac{1}{80} \frac{a^{2} U^{2}}{\kappa} .
\end{aligned}
$$

$$
S_{l}(\tau)=9 U^{2} \overline{\cos \theta \sin \theta \cos \phi \cos \theta^{\prime} \sin \theta^{\prime} \cos \phi^{\prime}},
$$

it is necessary to make some assumption about the statistical correlation between the values of $\phi$ in successive capillaries occupied by a marked particle. In the present paper, we shall assume that these are statistically independent, and also that the value of $\phi$ is independent of $\theta$. The contribution to $S_{l}(\tau)$ from particles which have changed capillaries in time $\tau$ is then identically zero, and hence

$$
S_{t}(\tau)=9 U^{2} \int_{0}^{1} \int_{0}^{1} \int_{0}^{2 \pi} \mu^{2}\left(1-\mu^{2}\right) \cos ^{2} \phi P\left(\xi_{0}, \mu, \tau\right) d \xi_{0} d \mu d \phi
$$

where $P\left(\xi_{0}, \mu, \tau\right)$ is given by $(4.7)$.

The assumption of the statistical independence of the values of $\phi$ in different capillaries is somewhat dubious when $U l / K \gg 1$ (as discussed in more detail in I). Roughly speaking, this is because the lateral meanderings of the streamlines are restricted by continuity requirements. However, the smaller is $U l / \kappa$, the better will be the assumption, since the capillaries by which a marked particle leaves a junction are equally likely if molecular diffusion is dominant.

$S_{l}(\tau)$ may be integrated with respect to $\tau$, giving

$$
\int_{0}^{\infty} S_{t}(\tau)=\frac{9 U^{2} l^{2}}{8} \int_{0}^{1} \mu^{2}\left(1-\mu^{2}\right) \frac{1}{D}\left(\frac{1}{M} \operatorname{coth} M-\frac{1}{M^{2}}\right) d \mu .
$$

Then $\kappa_{i}$ is the sum of (7.4) and (7.6). Altering the terms independent of $U$ so that $\kappa_{t}=\kappa_{m}$ when $U=0$ (for the same reasons as given in $\S 5$ ), we have

$$
\kappa_{l}=\kappa_{m}+\frac{1}{80} \frac{a^{2} U^{2}}{\kappa}+\frac{9 U^{2} l^{2}}{8} \int_{0}^{1} \mu^{2}\left(1-\mu^{2}\right) \frac{1}{D}\left(\frac{1}{M} \operatorname{coth} M-\frac{1}{M^{2}}\right) d \mu .
$$

It will be noticed that $\kappa_{l} \neq \kappa_{l}$, in accord with intuition and experience. 
When $U l / \kappa \ll 1$,

$$
\kappa_{t} \doteqdot \kappa_{m}+\frac{U^{2} l^{2}}{40 \kappa} \text {. }
$$

At the other extreme when $U l / \kappa \gg 1$, then

$$
\kappa_{t} \doteqdot \frac{3}{18} U l+\frac{1}{40} \frac{a^{2} U^{2}}{\kappa}+\kappa_{m}-\frac{\kappa}{3}+O\left(\frac{\kappa^{2}}{U l}\right) .
$$

The leading term of this expression agrees with that obtained by the random walk analysis.

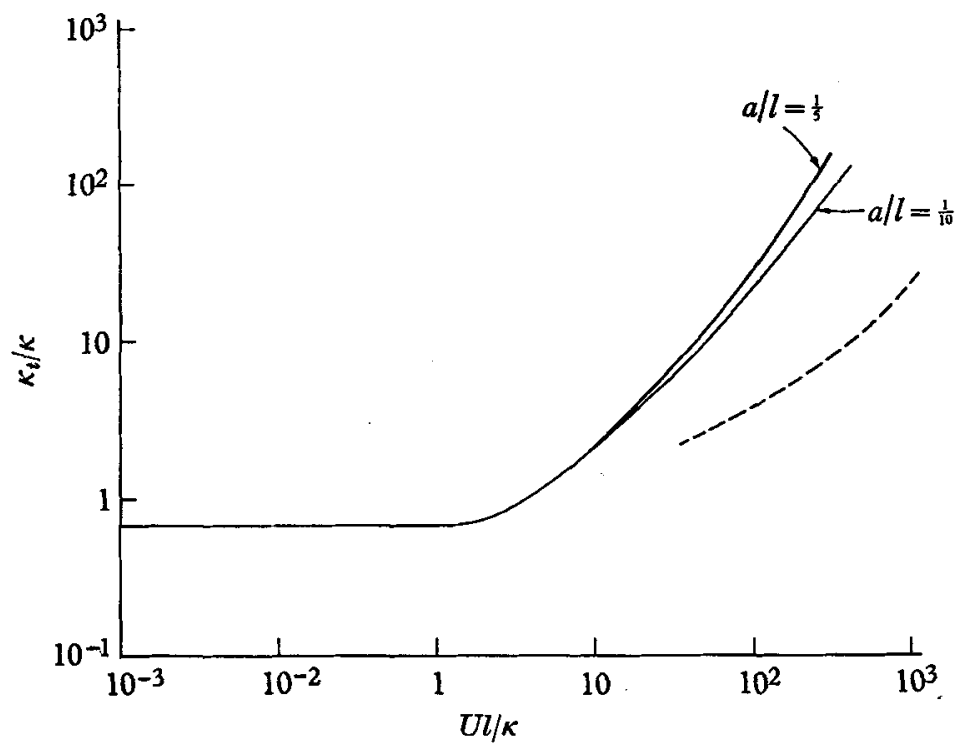

FiguRE 2. The lateral dispersion. - Calculated values with $\kappa_{m}=\frac{2}{3} \kappa$ and $a / l=\frac{1}{3}$ and $\frac{1}{10}$; ---, observed values (Hiby 1959).

The theoretical values with $\kappa_{m}=\frac{2}{3} \kappa$ and $a / l=\frac{1}{5}$ and $\frac{1}{10}$ are shown on figure 2 . Also shown are some measured values by Hiby (1959), in beds of spheres, for large values of $U l / \kappa$. The agreement is not good but a statistical correlation between successive values of $\phi$, consistent with a restricted lateral displacement of the streamlines, could reduce the calculated values of $\kappa_{t}$ considerably. The neglect of this correlation probably accounts for the differences between theory and experiment, and it is hoped to investigate this in later work.

\section{REFERENCES}

Anis, R. 1956 Proc. Roy. Soc. A, 235, 67.

Batchelor, G. K. \& Townsend, A. A. 1956 Article in Surveys in Mechanics (ed. G. K.

Batchelor and R. M. Davies), p. 352. Cambridge University Press.

Brackwalt, R. J., Rayne, J. R. \& Terry, W. M. 1959 J. Petrol Tech. $217,1$.

Carberry, J. J. \& Bretion, R. H. 1958 J. Amer. Inst. Chem. Engrs, 4, 367.

Hrвy, J. W. 1959 Paper presented at conference on displacement in porous media,

Imperial College, London, March 1959.

Saffman, P. G. 1959 J. Fluid Mech. 6, 321.

Taytor, G. I. 1921 Proc. Lond. Math. Soc. 20, 196.

Taylor, G. I. 1953 Proc. Roy. Soc. A, 219, 186.

Taylor, G. I. 1954 Proc. Roy. Soc. A, 225, 473.

Wooding, R. W. 1959 Proc. Roy. Soc. A, 252, 120. 